\title{
Development and Retranslational Validation of an In Vitro Model to Characterize Acute Infections in Large Human Joints
}

\author{
Ingo H. Pilz, Alexander Mehlhorn, David Dovi-Akue, Elia Raoul Langenmair, \\ Norbert P. Südkamp, and Hagen Schmal \\ Department of Orthopedic Surgery, University of Freiburg Medical Center, Hugstetter Straße 55, 79106 Freiburg, Germany \\ Correspondence should be addressed to Ingo H. Pilz; i.h.pilz@gmx.de
}

Received 3 September 2013; Accepted 9 April 2014; Published 30 April 2014

Academic Editor: Shigeru Kotake

Copyright (C) 2014 Ingo H. Pilz et al. This is an open access article distributed under the Creative Commons Attribution License, which permits unrestricted use, distribution, and reproduction in any medium, provided the original work is properly cited.

\begin{abstract}
Bacterial infections can destroy cartilage integrity, resulting in osteoarthritis. Goal was to develop an in vitro model with in vivo validation of acute joint inflammation. Inflammation in cocultivated human synovial fibroblasts (SFB), chondrocytes (CHDR), and mononuclear cells (MNC) was successively relieved for 10 days. Articular effusions from patients with $(n=7)$ and without $(n=5)$ postoperative joint infection in healthy patients (ASA 1-2) were used as model validation. Inflammation in vitro resulted in an enormous increase in IL-1 and a successive reduction in SFB numbers. CHDR however, maintained metabolic activity and proteoglycan synthesis. While concentrations of bFGF in vivo and in vitro rose consistently, the mRNA increase was only moderate. Concurring with our in vivo data, cartilage-specific IGF-1 steadily increased, while IGF-1 mRNA in the CHDR and SFB did not correlate with protein levels. Similarly, aggrecan (ACAN) protein concentrations increased in vivo and failed to correlate in vitro with gene expression in either the CHDR or the SFB, indicating extracellular matrix breakdown. Anabolic cartilage-specific BMP-7 with highly significant intra-articular levels was significantly elevated in vitro on day 10 following maximum inflammation. Our in vitro model enables us to validate early inflammation of in vivo cell- and cytokine-specific regulatory patterns. This trial is registered with MISSinG, DRKS 00003536.
\end{abstract}

\section{Introduction}

Acute inflammation, a rapid response to infectious microorganisms or injured tissue, culminates in the local recruitment and activation of immune cells. Goal is to resolve the initiating event by making microbes harmless or by removing cellular debris. In case of success, normal tissue architecture is restored by resolving acute inflammation. Otherwise, inflammatory processes will persist and evolve [1].

The most frequent endogenous causes in synovial human joints are hematogenic spread in the wake of a bacteremia [2] . These are mainly triggered by predisposing immunosuppressive factors such as diabetes or alcohol abuse in conjunction with bacteremia [3]. Cartilage, owing to its limited selfhealing capacity, is particularly vulnerable [4-7]. After just 6 days of inflammation, irreversible cartilage damage in a rabbit model was observed [8]. The reason for this is the impact on cartilage entailing destruction of the extracellular matrix (ECM) network. Degradation of the ECM, a specialized reservoir of regulatory factors, greatly influences the outcome of inflammatory responses $[9,10]$. The consequences are all the more serious as cartilage contains just $1-5 \%$ chondrocytes maintaining and regenerating ECM, and their amount declines with age [11-13]. Chondrocytes ensure equilibrium between the synthesis and degradation of their structural components such as collagen type II and proteoglycans. Therefore, the ECM's inflammation and destruction result in an altered chondrocytic phenotype with unrounding, increased proliferation, and altered gene expression [14]. The massive release of proinflammatory IL-1, during acute inflammation, for example, suffices to almost completely suppress collagen type I and II and aggrecan mRNA expression $[15,16]$. Altered collagen expression during this process results in "repair" cartilage with inferior biochemical properties [17]. 
We know very little about early events during acute bacterial inflammation in synovial joints. To elucidate these early events with respect to mediators and biochemical impacts on chondrocytes (CHDR), we established an in vitro coculture model comprising crucial cell types in synovial joints. We validated our model using the lavage fluids from acutely postoperatively infected knee joints as reference.

Inflammatory stimuli can be provoked by applying lipopolysaccharides (LPS) derived from gram-negative bacteria [18]. LPS is considered a potent activator of the macrophage secretory response [19]. They are, in addition to neutrophils, key mediators of the host's antimicrobial defence [20]. While LPS application results in a sharp and transient release of IL-1, it is quickly diluted during media exchanges. Phorbol 12-myristate 13-acetate (PMA) application, however, leads to stable, nontransient IL-1 accumulation [21]. Moreover, the PMA challenge shifts the ratio of IL-1 from intracellular towards extracellular accumulation, resulting in elevated IL-1 secretion [22]. These data suggest that LPS and PMA are ideal substances to modulate the period and severity of the inflammation and resultant responses and the pattern of expressed and secreted mediators, respectively.

Among the analyzed cytokines and mediators are the proinflammatory marker IL-1 [23] and aggrecan (ACAN). ACAN, a major constituent of the ECM [24, 25], effectively neutralizes compressive forces, and that together with an elevated intra-articular ACAN level has been associated with cartilage damage [26].

Furthermore, the following mediators were included in our analysis; bFGF with its remodeling role in damaged tissue (especially in cartilage) $[27,28]$, the anabolic and cartilage protective factor IGF-1 and the regenerative factor BMP-7 $[29,30]$. As these mediators were concomitantly determined in lavage fluids of acutely infected knee joints, our aim was to determine how reliably the in vivo situation can be reproduced in vitro. Can we determine early indicators in vitro and in vivo to improve diagnostic and therapy of joint infections, thus minimizing the need for surgery and helping to prevent cartilage damage at the same time?

\section{Material and Methods}

2.1. Isolation of Chondrocytes (CHDR). Femoral heads were obtained during hip arthroplasty operations following femoral neck fractures. The degrees of osteoarthritis (OA) were evaluated on X-rays using Croft's modification of the Kellgren and Lawrence grading system. Cells from patients with advanced $\mathrm{OA}$ ( $\geq$ grade 3 ) were not used for the experiments. Within 8 hours after surgery, the cartilage was separated from the bone, reduced to small pieces, and digested with collagenase CLS type II (Biochrom, Berlin, Germany) dissolved in Ham's F-12 medium with 10\% FBS (Invitrogen, Karlsruhe, Germany) and $1 \%$ penicillin/streptomycin (Invitrogen, Karlsruhe, Germany). Following a 16-hour digestion period, cells were filtered, washed, and prepared for the experiments. The cells' preparation had been approved by the Ethics Committee of the University of Freiburg as part of the "Tissue bank for research in the field of tissue engineering" project (GTE-2002).

2.2. Isolation of Synovial Fibroblasts (SFB). Synovia obtained during knee operations with an arthrotomy was kept buffered at $4^{\circ} \mathrm{C}$. Within 8 hours after surgery, synovia was minced in pieces measuring about $3 \times 3 \mathrm{~mm}$ followed by transfer into Ham's F-12 medium with 10\% FBS (Invitrogen, Karlsruhe, Germany), $1 \%$ penicillin/streptomycin (P/S, Invitrogen, Karlsruhe, Germany), and $1 \%$ collagenase P (Roche, Mannheim). The suspension was digested in an incubator for $16 \mathrm{~h}$ at $37^{\circ} \mathrm{C}$ and $5 \% \mathrm{CO}_{2}$. Liberated synovial fibroblasts (SFB) were filtered, washed, and seeded in expansion media (Ham's F-12, $10 \% \mathrm{FBS}, 1 \times \mathrm{P} / \mathrm{S})$.

\subsection{Isolation of Peripheral Blood Mononuclear Cells (MNC).} Human peripheral blood mononuclear cells were isolated from heparin-treated human whole blood by a Ficoll-Paque (Pharmacia, Piscataway, NJ, USA) gradient. The layer containing PBMCs was collected and washed three times with PBS. According to manufacturer's instructions, the cell population purified by this method consists of mainly lymphocytes and monocytes with little addition of granulocytes and erythrocytes.

\subsection{D Coculture}

2.4.1. CHDR Embedding into Alginate Beads. One million chondrocytes (CHDR) were resuspended in $1 \mathrm{~mL} 1.2 \%$ alginic acid (Sigma, Taufkirchen, Germany) and transferred into a syringe with a $27 \mathrm{G}$ needle (Braun, Melsungen, Germany). In order to polymerize stable CHDR/alginate beads, the cell suspension was dripped into $0.1 \mathrm{M} \mathrm{CaCl}_{2}$ solution. After 2 wash steps with $\mathrm{PBS}$, the beads were cultivated for one day in Ham's F-12, $10 \% \mathrm{FBS}, 1 \times \mathrm{P} / \mathrm{S}$, at $37^{\circ} \mathrm{C}, 5 \% \mathrm{CO}_{2}$.

2.4.2. SFB, CHDR, and MNC Culture. Two hundred thousand SFB per well in a 6-well plate were seeded and cultivated in Ham's F-12, $10 \% \mathrm{FBS}, 1 \times \mathrm{P} / \mathrm{S}$, at $37^{\circ} \mathrm{C}, 5 \% \mathrm{CO}_{2}$ for one day. The following day, around 20 alginate beads were given to the SFB per well. On the same day, freshly isolated MNC were adjusted to $1 \times 10^{6}$ cells per $\mathrm{mL}$ and placed in cell culture inserts with $1 \mu \mathrm{m}$ pore size (BD, Heidelberg, Germany) and cultivated for one day in Ham's F-12, $10 \%$ FBS, $1 \times \mathrm{P} / \mathrm{S}$, at $37^{\circ} \mathrm{C}, 5 \% \mathrm{CO}_{2}$ in 6 -well plates. On the following day, cell culture inserts were placed into the CHDR and SFB coculture. Over the following 3 days, we carried out a preconditioning phase of the coculture. With the start of the experiment on day 0 , the coculture was challenged with $250 \mathrm{ng} / \mathrm{mL}$ LPS (product number L6529, serotype $E$. coli. 055:B5, Sigma, Taufkirchen, Germany). On day 3, one half of the LPS challenged coculture was superchallenged by adding $100 \mathrm{ng} / \mathrm{mL}$ PMA (product number P1585, Sigma, Taufkirchen, Germany). On days 0, 3, and 7 half of the media was exchanged to minimize the inflammatory burden. This procedure was adapted to arthroscopic joint rinsing to minimize and halt the inflammatory burden. Media collected on days $0,3,7$, and 10 were preserved at $-80^{\circ} \mathrm{C}$. 
2.5. Clinical Evaluation. A consecutive series of 75 patients were recruited for the prospective collection of knee joint lavage fluid [31]. Inclusion criteria consisted of an arthroscopy of the knee joint because of an acute infection (confirmed macroscopically and microbiologically), informed consent to participate in the study, and aged between 18-70 years (MISSinG, DRKS 00003536). The 5 patients in the control group had no infection or cartilage lesions in MRI and diagnostic arthroscopy. Effusions were taken during arthroscopy and immediately kept frozen. Specimens were stored in liquid nitrogen until analysis. For model validation, 7 effusions were selected which fulfilled the conditions of a clearly defined starting point (all postoperative infections following arthroscopy) in basically healthy individuals (ASA 1 or 2). This setup ensured comparable conditions and a matching time frame for in vitro and in vivo analysis. The validation group's average age was 38 years \pm 16 years, with a male/female ratio of $57 / 43 \%$. The study was approved by the Ethics Board of the University of Freiburg (AN-EK-FRBRG-50/11). All patients participating in this study provided their written consent.

\subsection{Analyses of Synovial Joint Fluids and Coculture Supernatants}

2.6.1. ELISA. Cytokines and proteins as IL-1, bFGF, IGF-1, ACAN, and BMP-7 detected in synovial joint fluids from acutely infected knee joints and coculture supernatants were analyzed by ELISA (RnD, Minneapolis, MN, USA) according to manufacturer's instructions. Briefly, this assay employs the quantitative sandwich enzyme immunoassay technique. The microplate was precoated with a specific monoclonal antibody. Supernatants were applied to the wells and, after washing, an HRP-conjugated specific antibody was added to the wells. Following the next wash, color development was proportional to the protein concentration and was calculated by comparison with a standard.

2.6.2. Total Protein Determination. In order to normalize cytokine and mediators secreted in the synovial fluid as well as into the supernatant of the coculture, we divided cytokine and mediator concentrations by the amount of total protein present (TP) in synovial fluids and coculture supernatants using the Pierce BCA Protein Assay Kit (Thermo Scientific, Rockford, IL, USA). Briefly, standard and working reagents were prepared for microplate procedure as instructed by the manufacturer. Twenty-five microliters of standard and unknowns was pipetted into a 96-well microplate followed by adding $200 \mu \mathrm{L}$ to each well. The subsequent incubation at $37^{\circ} \mathrm{C}$ lasted 30 minutes $\left({ }^{\circ} \mathrm{C}=\right.$ degree Celsius $)$. Measurements were taken at $562 \mathrm{~nm}$ in a TECAN Infinite M200 Reader.

2.7. Real Time PCR (RT-PCR). RT-PCR was carried out for CHDR and SFB. RNA samples from days $0,3,7$, and 10 were transcribed into cDNA; RNA analysis was carried out for gene expression of aggrecan, IL-1, BMP-7, bFGF, and IGF1. Total mRNA was prepared using the Qiagen RNeasy kit according to manufacturer's instructions (Qiagen, Hilden, Germany). Total RNA (1 $\mu \mathrm{g})$ was treated with $1 \mathrm{U}$ DNAse I (Invitrogen, Karlsruhe, Germany) to remove genomic DNA. Poly-T primed cDNA synthesis was done using $1 \mathrm{U}$ reverse transcriptase III (RTIII, Invitrogen) per $1 \mu \mathrm{g}$ RNA according to manufacturer's instruction. TaqMan PCR assays were performed in 384-well plates in a Roche LightCycler480 (Roche, Mannheim, Germany) using the Roche LightCycler Mastermix. For gene expression analyses, Roche's universal ProbeLibrary Probes and recommended Universal ProbeLibrary Reference Gene Assays were used. The 2-step cycling conditions were denaturation at $95^{\circ} \mathrm{C}$ for $5 \mathrm{~min}$, followed by 45 amplification cycles at $95^{\circ} \mathrm{C}, 10 \mathrm{sec}, 60^{\circ} \mathrm{C}, 35 \mathrm{sec}$, and $72^{\circ} \mathrm{C}$ $1 \mathrm{sec}$. Data was quantified via $\Delta \Delta \mathrm{CT}$ comparisons. Data were normalized by comparing genes of interest versus reference genes (ACTB). Reaction efficiency is controlled by a relative standard curve and/or a calibrator per reaction.

\subsection{Histological Examinations}

2.8.1. CHDR. CHDR/alginate beads from days $0,3,7$, and 10 were preserved by fixation in PBS-buffered $2 \%$ formaldehyde for $24 \mathrm{~h}$ at room temperature, followed by fixation in $50 \mathrm{mM}$ $\mathrm{BaCl}_{2} / 1 \%$ sucrose and $1 \%$ eosin for 30 minutes. Afterwards, CHDR/alginate beads were dehydrated by graded dehydration in isopropyl alcohol (75\%, 96\%, and 100\%) for $10 \mathrm{~min}$ each, followed by paraffin embedding. To detect sulfated glycoproteins synthesized by CHDR, $5 \mu \mathrm{m}$ cuttings of CHDR/alginate beads were rehydrated in xylol and graded isopropyl alcohol (100\%, 96\%, and 75\%) steps for $10 \mathrm{~min}$ each. After a short rinse in $\mathrm{dH}_{2} \mathrm{O}$, incubation in $0.1 \mathrm{~N} \mathrm{HCl}$ for $10 \mathrm{~min}$ followed by incubation in $1 \%$ Alcian/Blue/ $0.1 \mathrm{~N} \mathrm{HCl}$ for $60 \mathrm{~min}$ took place. Counterstaining was carried out by nuclear fast red-aluminum sulfate solution $0.1 \%$ for $10 \mathrm{~min}$ followed by dehydration in graded isopropyl alcohol (75\%, $96 \%$, and $100 \%$ ) and xylol for 5 min each and embedding in Entellan (Sigma, Taufkirchen, Germany). Image acquisition and analysis were done using Zeiss Axioplan 200 and Axiovision 4.6 , respectively.

2.8.2. SFB. SFB grown on cover slides in coculture approaches were fixed by PBS-buffered $2 \%$ formaldehyde for $24 \mathrm{~h}$ at room temperature, followed by giemsa staining for $10 \mathrm{~min}$. Image acquisition and analysis were done by Zeiss Axioplan 200 and Axiovision 4.6, respectively. Cell numbers were evaluated by ImageJ (Wayne Rasband, NIH, http://imagej.nih.gov/ij/download). Colored pictures were converted to gray scale pictures. Background correction, scale adjustment, and watershed analysis (discrimination of adjacent cells) were carried out, followed by counting the numbers of particles larger than $50 \mu \mathrm{m}^{2}$. The correlation factor of 44.9 was obtained by dividing the sizes of growth area per well $\left(9.62 e-4 \mathrm{~m}^{2}\right)$ in a 6 -well plate by a factor through that of the section's image size $\left(2.14 e-5 \mathrm{~m}^{2}\right)$.

2.9. Data Analysis and Statistics. ELISA and TP were analyzed according to the manufacturer's instructions $(\mathrm{RnD}$, Minneapolis, MN, USA; Thermo Scientific, Rockford, IL, 
USA), creating a standard curve and reducing data using a four-parameter logistic (4-PL) curve fit by using GraphPad Prism 5 software (GraphPad Software, Inc., La Jolla, CA, USA). All values were expressed as mean \pm standard deviation. Data sets were examined with one- and two-way analysis of variance, and individual group means were then compared with the unpaired Student's $t$-test. In case of unequal variances, we applied the Aspin-Welch test. Since in vitro data did not reveal a normal distribution, statistical significance was nonparametrically tested using the $U$ test according to Mann and Whitney. Statistical significance was defined when $P<0.05$.

\section{Results}

3.1. Acute Inflammatory Challenge Results in Comparable Inflammatory Responses In Vivo and In Vitro. To determine inflammatory mediators expressed upon acute bacterial infection in large human joints, we set up a coculture model containing crucial articular human components. The inflammatory burden of the coculture challenged with single inflammatory stimuli was consecutively relieved on days 3,7 , and 10. This procedure mimics the applied clinical treatment of a repeated lavage. Mediators present in the supernatant were analyzed on the gene expression level as well.

3.2. Challenge with LPS and LPS/PMA Results in a Timely Regulated Response of the Proinflammatory IL-1. Inflammatory mediators formerly associated with altered cartilage biochemistry upon surgical [23] or chronic inflammation [32] were tested for their presence. In accordance with these findings, we observed significantly elevated inflammatory IL1 (Figure 1(a), infection $0.07 \pm 0.08$; control $2.6 \times 10^{-} \pm$ $4.2 \times 10^{-7}$ ) levels in synovial lavage fluids. Likewise, we noted significant increased IL-1 $(P<0.005)$ levels three days following the LPS challenge (Figure 1(b) $9.9 \times 10^{-9} \pm 0.5 \times$ $10^{-9}$; control $\left.0.6 \times 10^{-9} \pm 0.1 \times 10^{-9}\right)$. These concentrations remained at a significantly elevated level (PMA LPS, days 7$10, P<0.005,1.9 \times 10^{-8} \pm 0.1 \times 10^{-8}, 1.7 \times 10^{-8} \pm 0.5 \times 10^{-8}$; controls $7.1 \times 10^{-10} \pm 1.3 \times 10^{-10}, 3.4 \times 10^{-10} \pm 0.9 \times 10^{-10}$ ) which dropped slightly in LPS challenged samples (day 7 , $P<0.005,1.2 \times 10^{-8} \pm 0.2 \times 10^{-8}$; day $10, P<0.01$, $\left.1.0 \times 10^{-8} \pm 0.3 \times 10^{-8}\right)$. Regarding IL-1 mRNA expression, we detected a similar occurrence in the SFB and CHDR on day 3 (Figures 1(c) and 1(d)). We observed significantly elevated IL- $\beta$ gene expression in CHDR stimulated with LPS from day 3 onwards (Figure 1(c), days 3-7, $P<0.05$, $3.2 \pm 2.2,6.7 \pm 5.1$; day $10, P<0.01,7.5 \pm 4.3$; controls $0.20 \pm 0.05,0.08 \pm 0.08$, $0.1 \pm 0.1$ ). Gene expression following LPS PMA stimulation, however, peaked on day 7 and declined thereafter (Figure 1(c), days 3 and $10, P<0.05,25.3 \pm 24.0$; day $7, P<0.01$, $38.0 \pm 38.0)$. Significantly elevated IL-1 mRNA expression in SFB was observed upon LPS (Figure 1(d), days 7-10, $P<0.01$; $1.1 \pm 0.6,2.7 \pm 1.6$; controls $0.04 \pm 0.02,0.01 \pm 0.01)$ and the LPS PMA challenge (Figure $1(\mathrm{~d})$, days $7-10, P<0.05 ; 12.1 \pm 10.4$, $9.6 \pm 8.1)$.
3.3. The Homeostatic Cartilage Factor bFGF Senses Damage In Vivo as well as In Vitro. We included in this analysis the growth factor bFGF, known as an intrinsic cartilage repair factor upregulated upon initial cartilage damage $[27,33]$. We detected an increased bFGF level in lavage fluids (Figure 2(a), infection $8.3 \times 10^{-2} \pm 1.3 \times 10^{-1}$; control $7.9 \times 10^{-3} \pm 1.4 \times 10^{-2}$ ) and the coculture which persisted at a significantly elevated level from day 3, continuing through the coculture period (Figure 2(b), LPS, day 3, $P<0.01,5.3 \times 10^{-8} \pm 1.5 \times 10^{-8}$; day $7, P<0.001,4.8 \times 10^{-8} \pm 0.3 \times 10^{-8}$; day $10, P<0.05$, $1.1 \times 10^{-7} \pm 0.5 \times 10^{-7}$; LPS PMA, days $3-7, P<0.01$, $5.3 \times 10^{-8} \pm 1.5 \mathrm{E}-8,1.9 \times 10^{-7} \pm 0.4 \mathrm{E}-7$; day $10, P<0.005$, $2.7 \times 10^{-7} \pm 0.5 \times 10^{-7}$; controls $1.1 \times 10^{-8} \pm 0.2 \times 10^{-8}$, $\left.9.3 \times 10^{-8} \pm 0.5 \times 10^{-8}, 1.2 \times 10^{-8} \pm 0.6 \times 10^{-8}\right)$. This is also reflected on gene expression in CHDR and SFB (Figures 2(c) and $2(\mathrm{~d})$ ). Correspondingly, from day 3 on, we noted a $3-$ 5 -fold rise in mRNA expression in LPS-stimulated CHDR. Similarly, a 3-5-fold increase in gene expression was observed in SFB treated with LPS on days 7 and 10 (Figure 2(d), day 10, $P<0.05,0.4 \pm 0.2$; control $0.07 \pm 0.05)$. Gene expression in CHDR challenged with LPS PMA exhibited a circa 5-fold upregulation on days 3 and 7 (Figure 2(c), LPS PMA, day 7, $P<0.05,24.8 \pm 19.9$; control $5.5 \pm 4.6)$. Even higher gene expression ratios were observed in SFB stimulated with LPS PMA on days 7 and 10 (Figure $2(\mathrm{~d}), P<0.05,1.3 \pm 0.4$, $1.3 \pm 1.0$; controls $0.16 \pm 0.18,0.07 \pm 0.05)$.

3.4. Rise in the Anabolic and Protective Factor IGF-1 Correlates with Increasing Damage. IGF-1 synthesized by many cells of mesenchymal origin is a major metabolic growth factor in articular cartilage $[34,35]$. In addition, IGF-1 is able to lower the degradation of proteoglycan in cartilage exposed to IL1 and TNF $\alpha$ [36]. We therefore included IGF-1 in this study to evaluate the effects of acute bacterial inflammation on IGF-1 expression. While we detected low IGF-1 levels in the lavage fluids of healthy patients, these levels were significantly elevated in infected joints (Figure 3(a), $P<0.05$, infection $0.2 \pm 0.1$; control $7.5 \times 10^{-3} \pm 1.0 \times 10^{-2}$ ).

In the coculture, we observed significant increased IGF1 level after challenge with LPS PMA (Figure 3(b), days 7 and $10, P<0.01 ; 4.8 \times 10^{-8} \pm 0.6 \times 10^{-8}, 7.6 \times$ $10^{-8} \pm 0.9 \times 10^{-8}$; controls $2.8 \times 10^{-8} \pm 0.4 \times 10^{-8}, 3.5 \times$ $10^{-8} \pm 0.7 \times 10^{-8}$ ). Despite different gene expression levels having been observed, CHDR expression did not result in a statistically significant upregulation upon inflammatory challenge (Figure 3(c)). However, we did observe significant different IGF-1 mRNA expression (around 6-fold) in the SFB after 10 days (Figure $3(\mathrm{~d}), P<0.05,0.7 \pm 0.1$; control $0.1 \pm 0.1$ ).

3.5. Elevated Liberation of Aggrecan following Acute Inflammation In Vivo and In Vitro. An elevated intra-articular ACAN level, formerly associated with cartilage damage [26], was detected in our analyses as well. Significantly elevated ACAN levels (Figure 4(a), around 30-fold, $P<0.01$, infection $3.4 \pm 2.2$; control $0.1 \pm 0.1$ ) were observed in the lavage fluids from infected knee joints. We noted significantly elevated ACAN levels (3-fold) in the supernatants of the coculture after concerted application of LPS and PMA towards the end 


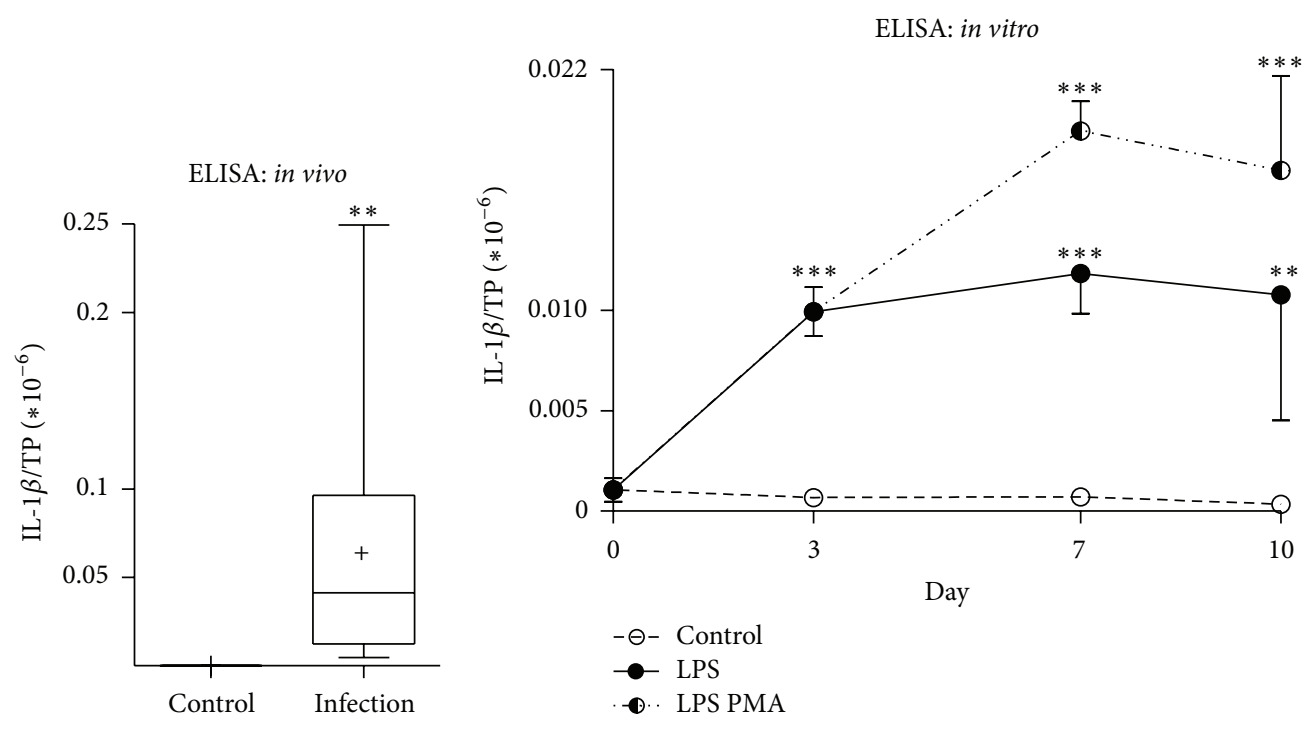

(a)

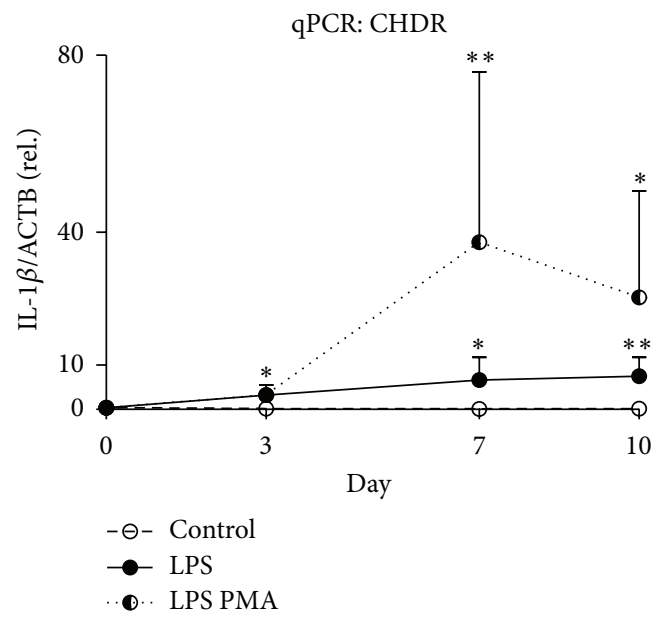

(c) (b)

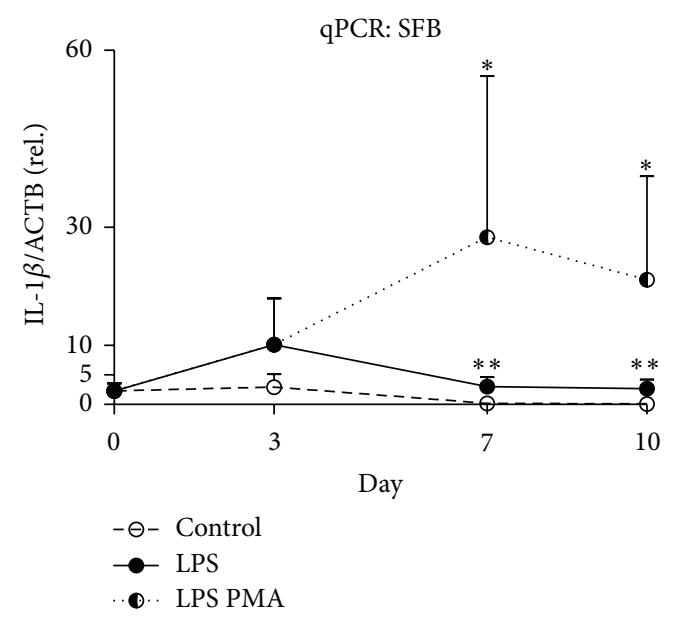

(d)

FIGURE 1: Detecting the inflammatory cytokine IL-1 in in vivo and in in vitro samples. Significantly elevated IL-1 level was detected by ELISA in lavage fluids from acutely infected knee joints $\left((\mathrm{a})^{* *}\right)$ as well as in cell culture supernatants upon an LPS challenge ((b) days $3,7^{* * *}$, day $\left.10{ }^{* *} P<0.01\right)$ or LPS PMA ((b) days $\left.7-10,{ }^{* * *}\right)$. Accordingly, mRNA expression increased significantly in CHDR ((c) LPS: days $3,7^{*}$, day $10^{* *}$, LPS PMA: day $7^{* *}$, day $\left.10^{*}\right)$ and in SFB ((d) LPS: days 7, $10^{* *}$, LPS PMA: days 7, $\left.10^{*}\right)$. Statistics: (a) Mann-Whitney test and (b)-(d) Student's $t$-test, ${ }^{*} P<0.05,{ }^{* *} P<0.01$, and ${ }^{* * *} P<0.005$. ELISA data were normalized versus total protein content (TP).

of the cultivation period (Figure 4(b), LPS PMA, $P<0.05$, $1.6 \times 10^{-6} \pm 0.5 \times 10^{-6}$; control $\left.5.4 \times 10^{-7} \pm 0.5 \times 10^{-7}\right)$. The ACAN gene expression persisted throughout the observation period but the expression pattern did not correlate with increased ACAN protein levels (Figures 4(c) and $4(d))$.

3.6. Cartilage Metabolic and Protective Factor BMP-7 Strongly Induced after Profound and Sustained Damage In Vivo and In Vitro. Osteogenic protein 1 (OP-1) or BMP-7, which is strongly associated with cartilage metabolism and boosts ECM synthesis, has the capacity to repair damaged cartilage $[29,37]$. We noted significantly elevated BMP-7 levels in both lavage fluids (Figure 5(a), $P<0.01$, infection $3.2 \times$ $10^{-8} \pm 3.0 \times 10^{-8}$, control $1.8 \times 10^{-11} \pm 4.0 \times 10^{-11}$ ) from infected joints and in vitro. Maximal inflammatory challenge with LPS and PMA resulted in significantly elevated BMP7 levels on day 10 (Figure 5(b), LPS PMA, $P<0.01$, $2.3 \times 10^{-11} \pm 0.2 \times 10^{-11}$; control n.d.). In concert with an increased BMP-7 protein level, SFB challenged with LPS or LPS PMA manifested a rise in gene expression (Figure 5(c)) of 2.5-fold (LPS, day 7, $P<0.05,1.1 \times 10^{-4} \pm 0.6 \times 10^{-4}$; control $4.6 \times 10^{-5} \pm 3.4 \times 10^{-5}$ ) and 7-fold (LPS PMA, day $10, P<0.05,3.8 \times 10^{-4} \pm 1.4 \times 10^{-4}$; control $0.5 \times$ $\left.10^{-4} \pm 0.3 \times 10^{-4}\right)$. We detected no BMP-7 gene expression in CHDR. 


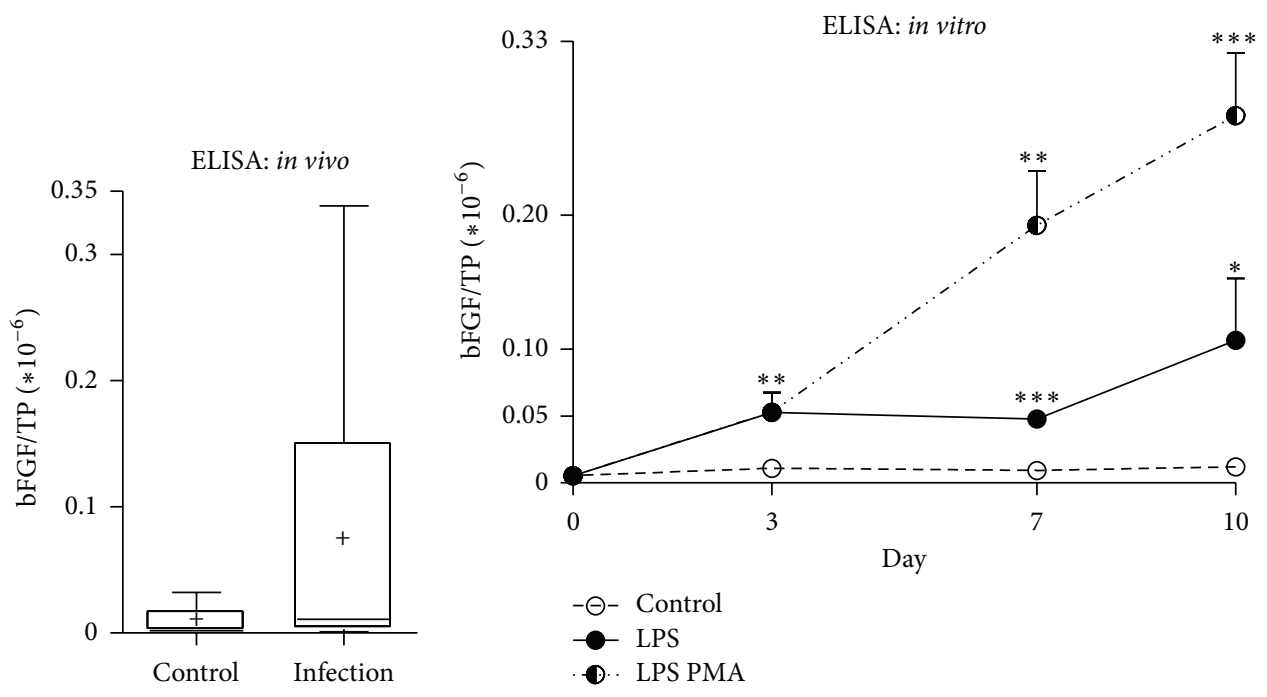

(a)

(b)

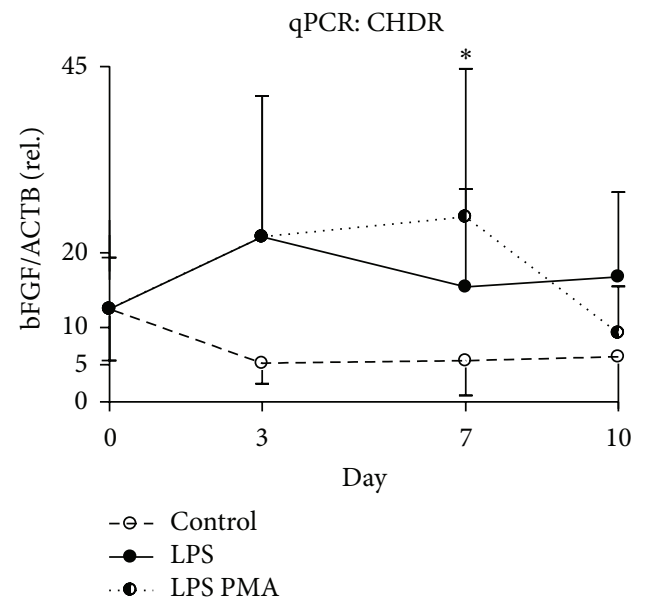

(c)

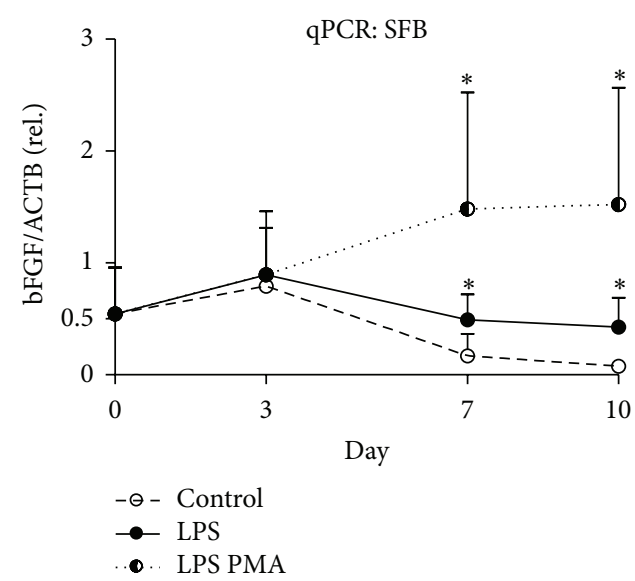

(d)

FIGURE 2: Detecting bFGF in in vivo and in vitro samples. Elevated bFGF levels observed in lavage fluids from acutely infected knee joints (a) which were significantly elevated in cocultures challenged by LPS ((b) day $3^{*}$, day $7^{* * *}$, and day $\left.10^{*}\right)$ or LPS PMA ((b) day $7^{* *}$, day $\left.10^{* * *}\right)$, too. Increased expression of bFGF mRNA was detected in CHDR and SFB upon LPS and LPS PMA challenge (c, d). Statistics: (a) Mann-Whitney test and (b)-(d) Student's $t$-test, ${ }^{*} P<0.05,{ }^{* *} P<0.01$, and ${ }^{* * *} P<0.005$. ELISA data were normalized versus total protein content (TP).

3.7. CHDR Remain Viable and Retain Ability to Synthesize Proteoglycans. Chondrocytes, the cells that synthesize and maintain cartilage, were analyzed for their ability to resist potentially damaging effects by maintaining their viability and functionality. For this, metabolic activity (by converting the MTS tetrazolium salt into formazan) was used to evaluate the viability of CHDR after an LPS and LPS PMA challenge (Figure 6(a)). Compared to controls, LPS-treated CHDR tend to increase their metabolic activity. LPS PMA-treated CHDR however exhibit diminished metabolic activity over time. Altogether, we observed no significant differences in viability and metabolic activity throughout the experimental procedure. With respect to functionality, CHDR were subject to Alcian-Blue staining for chondroitin sulphate (the major cartilage component in aggrecan) [13]. Compared to the controls (Figure 6(b), left), neither LPS (Figure 6(b), middle) nor LPS PMA (Figure 6(b), right) challenged CHDR lost their ability to produce sulfated proteoglycans at the end of the cultivation period.

3.8. Higher Inflammatory Challenge Causes a Decline in SFB Numbers. While SFB numbers in control assays increased continuously, we noted a significant decrease in cell numbers in the LPS and LPS PMA challenged assays (Figure 7(a)). Whereas the number of SFB challenged with LPS exhibited a significant decline on day 10 (control $3.1 \times 10^{5} \pm 2.6 \times 10^{4}$; LPS $1.6 \times 10^{5} \pm 4.6 \times 10^{4}$; LPS PMA $1.4 \times 10^{5} \pm 5.2 \times 10^{4}$ ), LPS PMA challenged SFB led to a significant decline in SFB numbers already from day 7 on (control $2.5 \times 10^{5} \pm 3.2 \times 10^{4}$; LPS PMA 


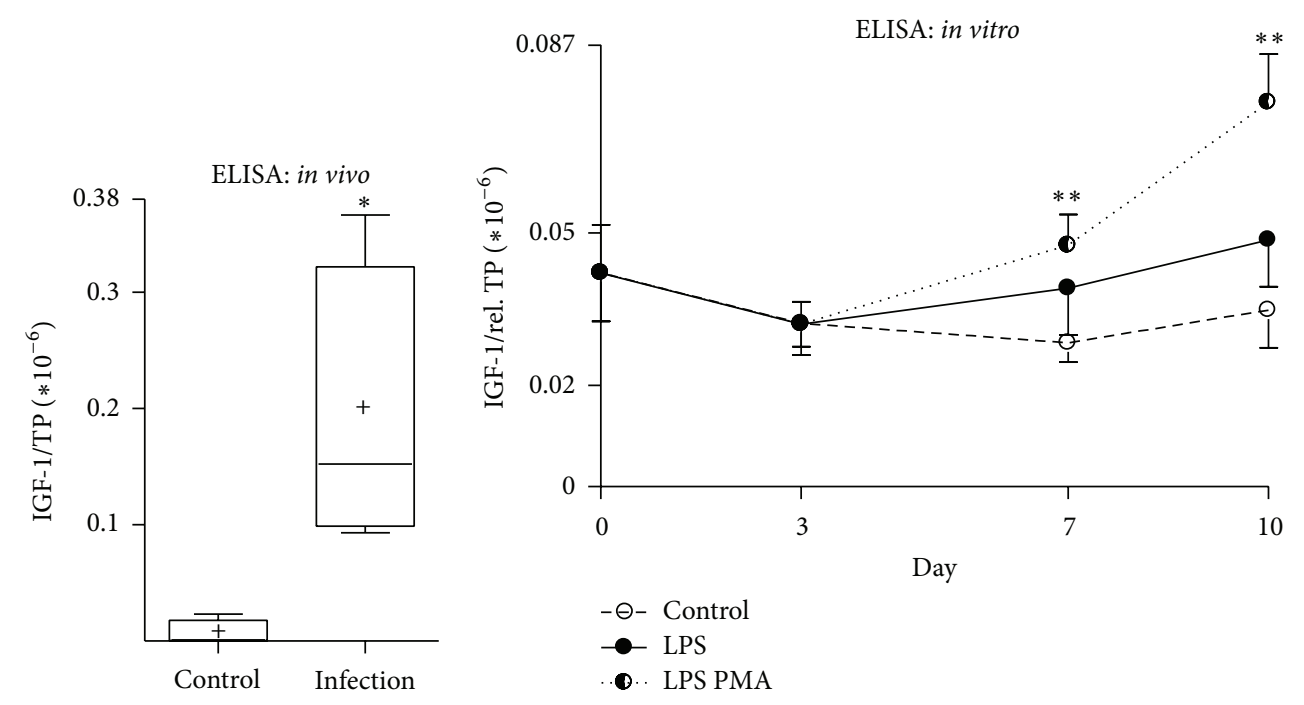

(a)

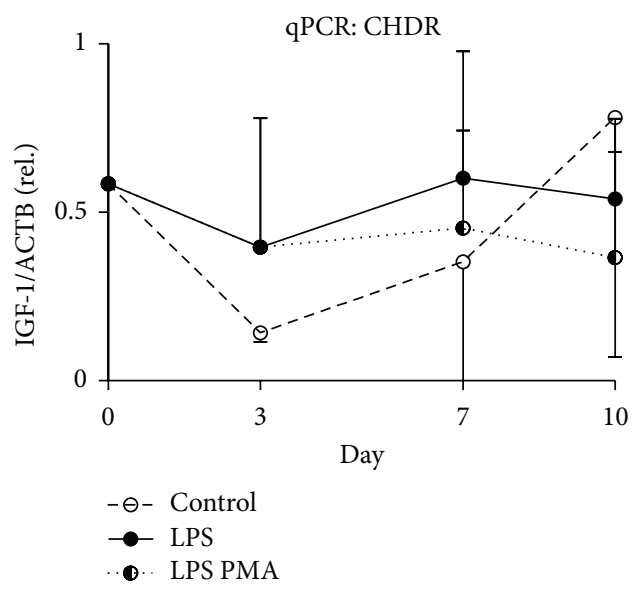

(c) (b)

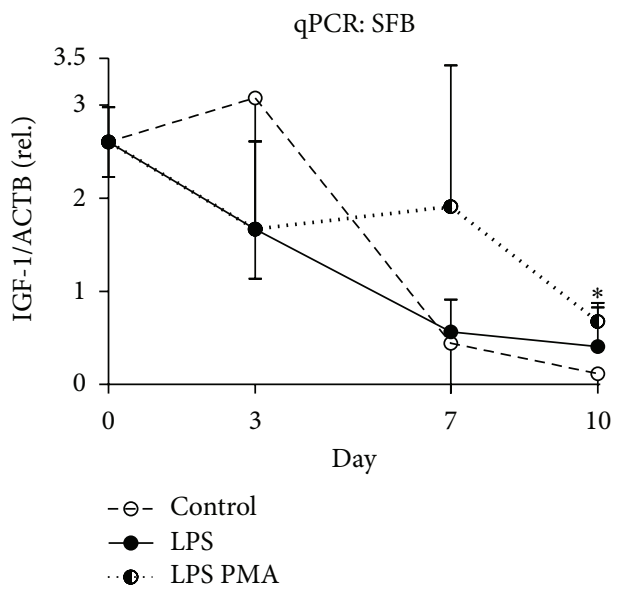

(d)

FIGURE 3: Detecting IGF-1 in in vivo and in vitro samples. Lavage fluids from acutely infected knee joints reveal elevated IGF-1 levels (a) also significantly elevated in cocultures challenged by LPS PMA ((b) days 7-10 **). No significantly altered IGF-1 mRNA expression in CHDR was apparent (c). After prolonged exposure to LPS PMA, however, significantly increased expression of IGF-1 mRNA was detected in SFB ((d) day $10^{*}$ ); (a) Mann-Whitney test and (b)-(d) Student's $t$-test, ${ }^{*} P<0.05,{ }^{* *} P<0.01$, and ${ }^{* * *} P<0.005$. ELISA data were normalized versus total protein content (TP).

$\left.1.6 \times 10^{5} \pm 2.5 \times 10^{4}\right)$. This was confirmed by histological SFB findings on day 10 .

\section{Discussion}

We developed and validated an in vitro model to reproduce the effects of acute inflammation in large human joints. The advantage of our model consists in monitoring impacts following acute inflammation over an extended time course including concurrent protein and its corresponding gene expression. The model compensates for the lack of concordance between protein and mRNA data. We observed that, after an initial release of proinflammatory IL-1 and catabolic bFGF, a delayed counterregulation of protective anabolic factors as BMP-7 takes place. We were thus able to demonstrate that both CHDR and SFB produce cartilagespecific mediators.

Longer-lasting inflammation inevitably results in a loss of cartilage integrity involving impaired joint function and ultimate destruction $[38,39]$. Proinflammatory macrophage derived IL-1 [40] manifested significantly elevated levels in the synovial fluids of affected patients. The coculture reflected this as well. Here, we detected significantly elevated IL-1 levels following LPS and LPS PMA shots throughout the cultivation period. Superimposition of PMA to LPSstimulated cocultures resulted in enhanced IL-1 secretion [22]. Whereas LPS primarily causes a sharp and transient release of IL-1, there is a longer-lasting response to PMA [21]. These two substances enabled us to modulate the severity of the inflammatory stimulus, especially regarding the deleterious effects on CHDR. In a clinical context, the 


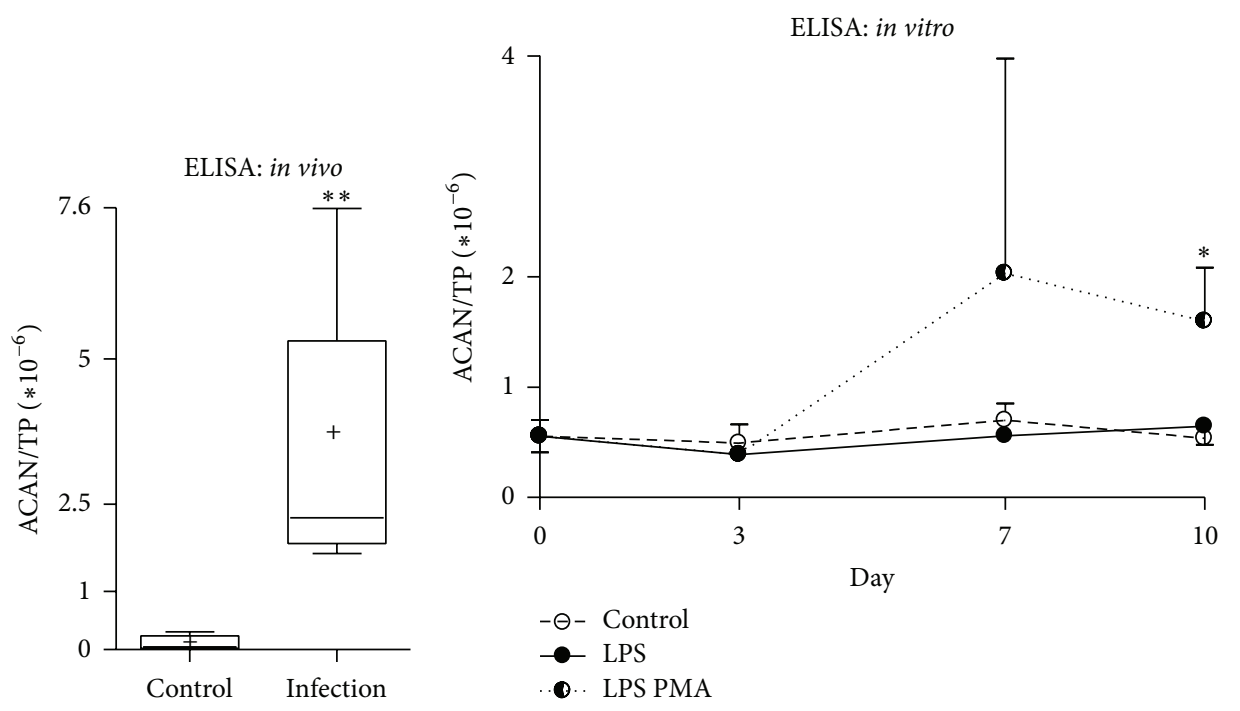

(a)

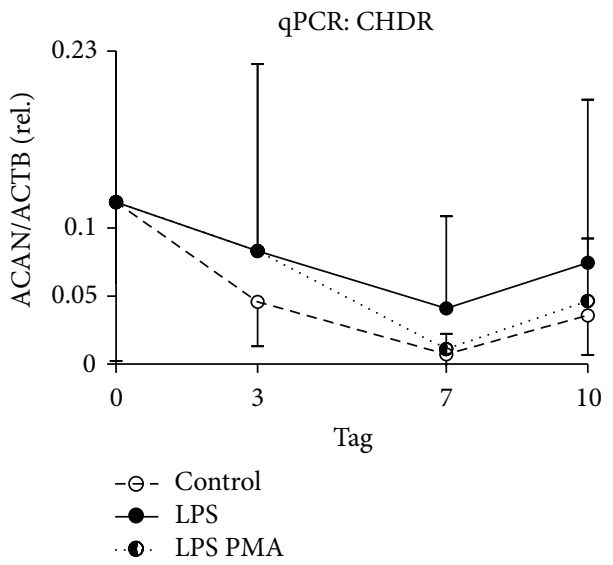

(c) (b)

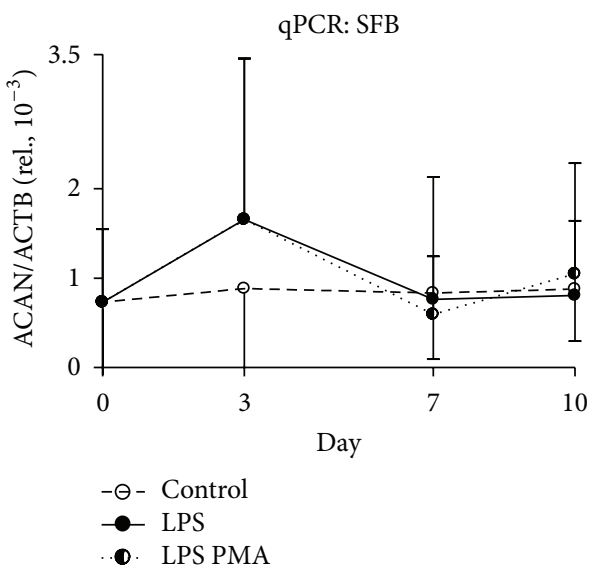

(d)

FIGURE 4: Detecting aggrecan in in vivo and in vitro samples. Significantly increased aggrecan (ACAN) levels were detected by ELISA in lavage fluids from acutely infected knee joints $\left((\mathrm{a})^{* *}\right)$. This was likewise observed in cocultures after prolonged LPS PMA challenge $((\mathrm{b})$ day $\left.10^{*}\right)$. LPS and LPS PMA challenge results in slightly increased ACAN mRNA expression in CHDR ((c) days 3-10) and SFB ((d) day 3). Statistics: (a) Mann-Whitney test and (b)-(d) Student's $t$-test, ${ }^{*} P<0.05$ and ${ }^{* *} P<0.01$. ELISA data were normalized versus total protein content (TP).

extent of inflammation was determined by microbiologically analyzing lavage fluids.

Interestingly, the inflammatory stimulus was also apparent in an altered gene expression. We noticed significantly elevated IL-1 gene expression levels in CHDR and SFB. A further and long-lasting increase in both cell types was observed following PMA application. These results demonstrate that CHDR and SFB are not only affected by inflammation. Moreover, they actively support and maintain inflammation and thus can lead to remodulation [41] and finally destruction [42] of the cartilage. Bivalent effects of IL-1 on human CHDR matrix degradation have been reported elsewhere [41].

Elevated catabolic bFGF, associated with disturbed cartilage homeostasis [27, 43], was present in lavage fluids, which were significantly elevated in vitro following LPS and PMA challenge. This occurred successively and in a manner correlating with the amount of damage. It is conceivable that altered homeostasis culminates in remodelling processes in CHDR and SFB. Equally, following LPS and PMA stimuli, bFGF gene expression in CHDR increased up to fourfold, and about 20 -fold in SFB.

Similar observations revealed that the ectopic expression of bFGF and EGF in CHDR resulted in increased proliferation and a concomitant decrease in collagen type II [33] and aggrecan [44] expression. A major function of CHDR, however, consists of ensuring cartilage homeostasis. But, differentiated CHDR possess a very limited capacity to proliferate. So, inducing proliferation [45] by enhancing bFGF synthesis might limit damaging effects.

Concomitant with increased bFGF levels, we noted significantly elevated IGF-1 levels in synovial lavage fluid as well as in LPS PMA challenged cocultures. Reports have shown 


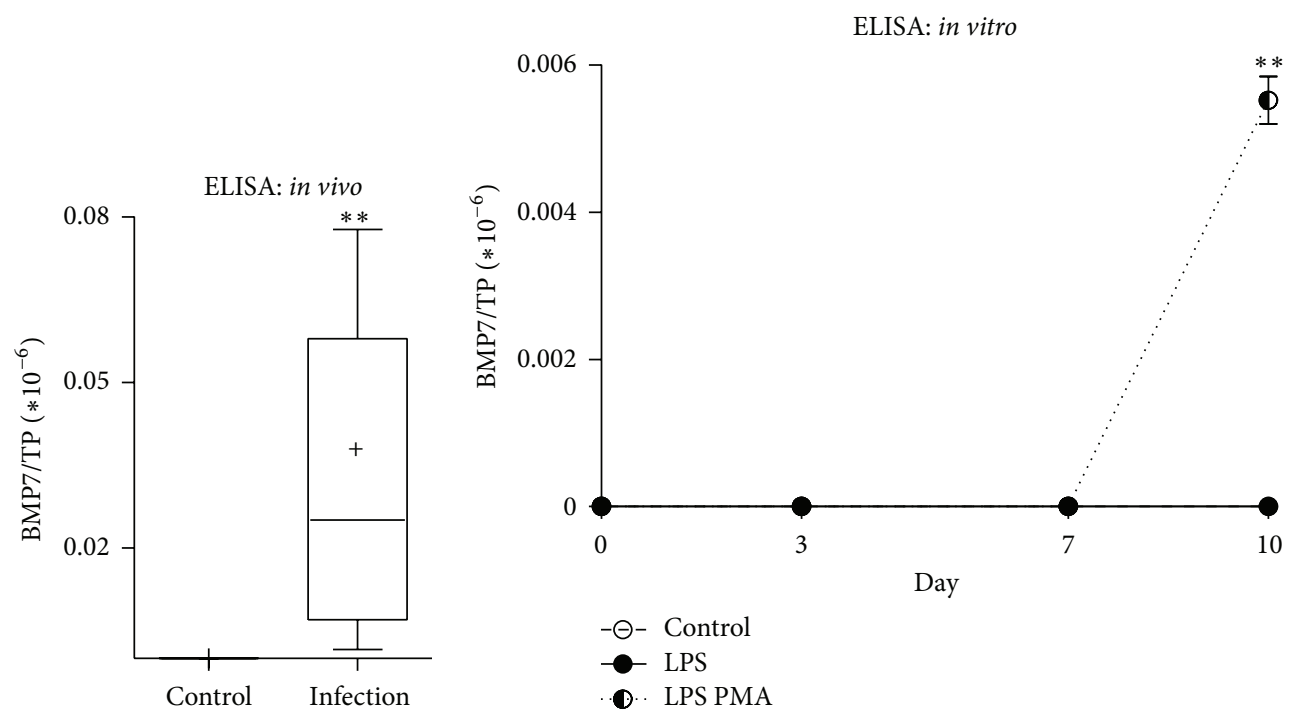

(a)

(b)

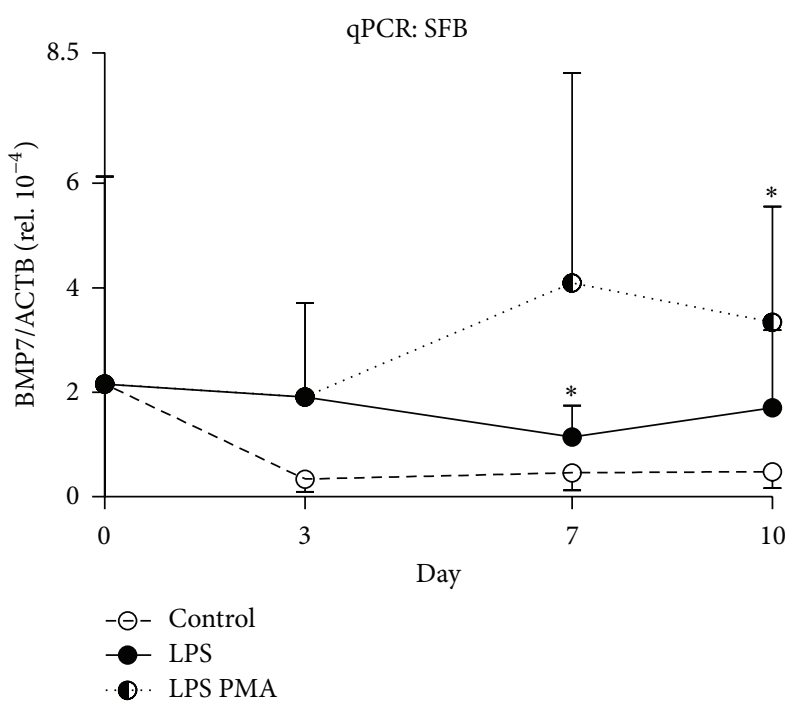

(c)

FIGURE 5: Detecting BMP-7 in in vivo and in vitro samples. Significantly elevated BMP-7 levels were detected by ELISA in lavage fluids from acutely infected knee joints $\left((\mathrm{a}){ }^{* *}\right)$ also observed in cocultures after prolonged challenge with LPS PMA $\left((\mathrm{b})\right.$ day $\left.10^{* *}\right)$. While no BMP-7 mRNA expression in CHDR was detected ((c) n.d.), a significant increase in BMP-7 mRNA expression was observed in SFB after either an LPS ((c) day $7^{*}$ ) or LPS PMA challenge ((c) day $\left.10^{*}\right)$. Statistics: (a) Mann-Whitney test and (b), (c) Student's $t$-test, ${ }^{*} P<0.05$ and ${ }^{* *} P<0.01$. ELISA data were normalized versus total protein content (TP).

that IGF-1 induces cartilage-specific induction of matrix synthesis [35] and prevents cytokine-stimulated degradation of proteoglycan in cartilage [36] to a certain extent. Supposedly, the progressive release of IGF-1 from the CHDR surrounding ECM into coculture supernatant is a means of antagonizing these effects. Thus, a stress-induced release of IGF-1 from the ECM precedes active counterregulation on gene expression level. An indication of this might be the fact that IGF1 gene expression detected in CHDR and SFB did not correlate with protein secretion. Only over the long term did $\mathrm{SFB}$, exposed to a stronger challenge, exhibit a significantly elevated IGF-1 mRNA level. An explanation for this may be either a regulatory mechanism or differences between IGF-1 gene expression and protein synthesis, as shown previously. On the other hand, SFB might contribute to minimizing deleterious effects when necessary. Nevertheless, a net loss of proteoglycan and the suppression of beneficial IGF-1 effects may occur. High bFGF levels were reported to shift the finetuning capabilities of cartilage to support ECM homeostasis versus a catabolic situation [28].

In this context we find the explanation for the significantly elevated levels of the proteoglycan ACAN detected 


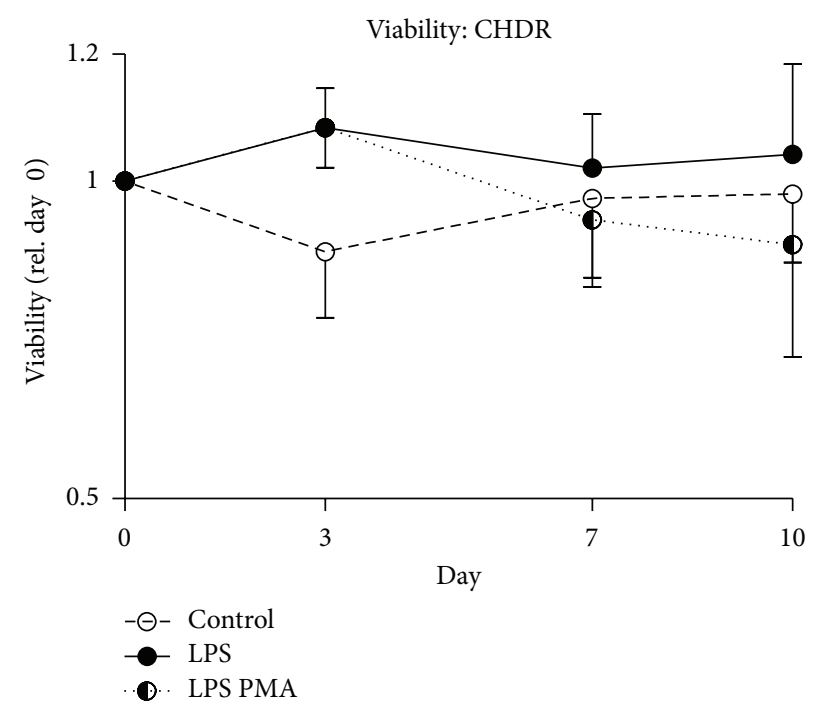

(a)

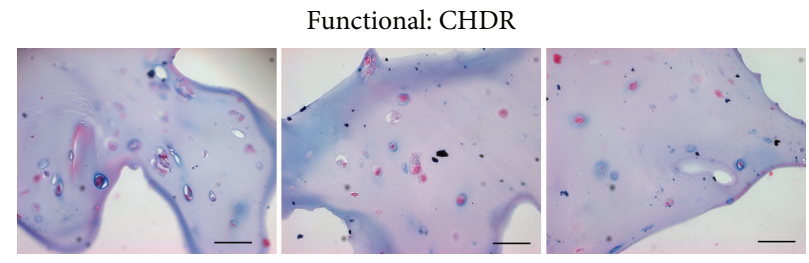

(b)

FIGURE 6: Viability and functionality of chondrocytes (CHDR). Cocultivated CHDR exposed to LPS, LPS PMA, or unchallenged were tested for their viability by MTS (a). While the LPS challenge tends to increase metabolic activity in CHDR, prolonged and severe inflammatory challenge by LPS PMA tend to result in diminished metabolic activity. However, we observed no significant differences in viability (a). Histological analyses by Alcian-Blue $\mathrm{HCl}$ staining for highly sulphated glycoproteins support these findings ((b) left: control; middle: LPS; right: LPS PMA challenge). Statistics: (a) Student's $t$-test. Scale bar $50 \mu \mathrm{m}$.

in lavage fluids from acutely infected knee joints. Similarly, concerted application of LPS and PMA resulted in a threefold increase in ACAN in supernatants of the cocultures as well. In line with our observations, the liberation of total or spliced ACAN following matrix breakdown during osteoarthritis progression has been described ACAN gene expression persisting throughout the experiment in CHDR and SFB did not correlate with an increased ACAN protein level. The disparate ACAN protein and gene expression may reflect a different regulatory mechanism. Or it might simply be evidence of uncontrolled liberation of ACAN owing to devastating effects during a massive inflammation as provoked by concerted LPS and PMA application.

The significantly elevated presence of the proanabolic [46] cartilage repair factor BMP-7 [29, 30] in the lavage fluids might indicate a repair attempt. In this regard, there is evidence that BMP-7 antagonizes the suppressive effects of LPS [47]. Furthermore, we observed significant BMP-7 levels in our cocultures after maximal inflammatory stimulus. Here, a significantly elevated BMP-7 level on day 10 might reflect the aforementioned situation. In addition, BMP-7 gene expression observed in SFB significantly rose in the course of inflammation caused by both LPS and LPS PMA. This in turn might also indicate that SFB or synovia assumes a repair function if, for example, CHDR are overwhelmed. During early events of an acute inflammation, a specific cytokine pattern in the joint cleft could initiate such a rescue operation to help cartilage to fend off damaging effects. For example, the exogenous addition of BMP-7 prevented cartilage loss and resulted in histological proof of equal amounts of this factor in cartilage and synovium $[48,49]$.

CHDR in cocultures retained their viability and functionality throughout the experiment. Whereas we observed a slight increase in metabolic activity after LPS stimulus, the addition of LPS PMA led to a gradual decline. In addition, Alcian-Blue staining confirmed that CHDR were capable of maintaining the synthesis of sulphated proteoglycans. Challenged SFB, however, decreased in cell numbers.

\section{Conclusion}

Our in vitro model enabled us to successfully monitor acute inflammatory effects on crucial components such as CHDR and SFB in large synovial joints over an extended period of time. By examining the synovial lavage fluids from acutely infected knee joints we were able to verify these results. In addition, by reproducing early inflammatory events on 


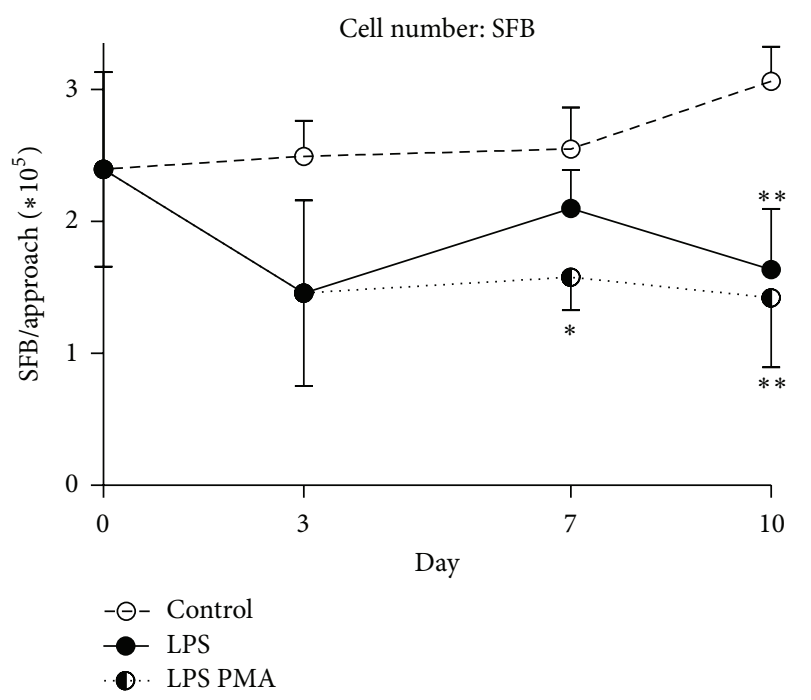

(a)

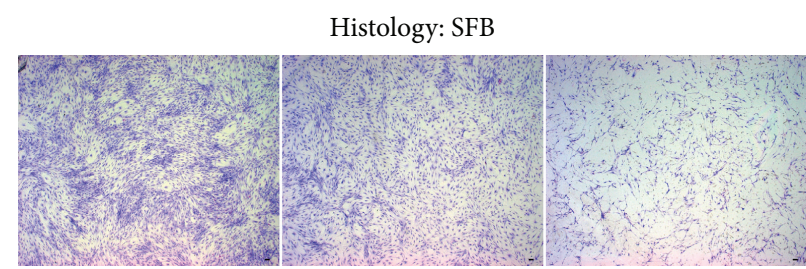

(b)

FIgURE 7: Synovial fibroblasts (SFB) proliferation. Originally in all cocultures, $2 * 10^{5} \mathrm{SFB}$ were seeded per well. While the SFB numbers increased steadily in controls, cell numbers in LPS decreased significantly (day $10,{ }^{* *} P<0.01$ ) and LPS PMA assays (day $7,{ }^{*} P<0.05$; day $10,{ }^{* *} P<0.01$ ). This observation is supported by giemsa-stained SFB on day 10 (b, left: control; middle: LPS; right: LPS PMA challenged cocultures). Statistics: (a) Student's $t$-test, ${ }^{*} P<0.05,{ }^{* *} P<0.01$; cell number was determined by ImageJ. Scale bar $100 \mu \mathrm{m}$.

protein and gene expression levels, we can draw conclusions regarding early inflammatory situations in vivo. Moreover, we provide evidence that SFB express cartilage protective factor BMP-7 to counteract detrimental inflammatory effects.

\section{Conflict of Interests}

The authors declare that they have no competing interests.

\section{Authors' Contribution}

Study conception and design were carried out by Hagen Schmal, Ingo H. Pilz, and Norbert P. Südkamp. Acquisition of data was done by Ingo H. Pilz, Alexander Mehlhorn, David Dovi-Akue, Elia Raoul Langenmair, Norbert P. Südkamp, and Hagen Schmal. Analysis and interpretation of data were done by Ingo H. Pilz, Alexander Mehlhorn, David Dovi-Akue, Elia Raoul Langenmair, Norbert P. Südkamp, and Hagen Schmal. All of the authors were involved in drafting the paper or revising it critically for important intellectual content, and all of the authors approved the final version to be submitted for publication. The authors had full access to all of the data in the study and take responsibility for the integrity of the data and the accuracy of the data analysis.

\section{Acknowledgments}

This study was supported by the Bundesministerium für Bildung und Forschung (01EC1001D, Verbund: Muskuloskelettale Infektionen (MISSinG): Teilprojekt 4). The article processing charge was funded by the open access publication fund of the Albert Ludwigs University Freiburg.

\section{References}

[1] J. S. Pober and W. C. Sessa, "Evolving functions of endothelial cells in inflammation," Nature Reviews Immunology, vol. 7, no. 10, pp. 803-815, 2007.

[2] H.-G. Simank, B. Wadi, and L. Bernd, "Joint empyema," Der Orthopade, vol. 33, no. 3, pp. 327-331, 2004.

[3] M. Diefenbeck, D. Abitzsch, and G. O. Hofmann, "Joint infections. Known facts and new trends," Der Unfallchirurg, vol. 115, no. 6, pp. 489-495, 2012.

[4] J. A. Buckwalter, "Articular cartilage: injuries and potential for healing," The Journal of Orthopaedic and Sports Physical Therapy, vol. 28, no. 4, pp. 192-202, 1998.

[5] J. A. Buckwalter and J. Martin, "Degenerative joint disease," Clinical symposia, vol. 47, no. 2, pp. 1-32, 1995.

[6] E. B. Hunziker, "Articular cartilage repair: basic science and clinical progress. A review of the current status and prospects," Osteoarthritis and Cartilage, vol. 10, no. 6, pp. 432-463, 2002. 
[7] K. P. Gunther, H. P. Scharf, and W. Puhl, "Standardization of roentgen diagnosis in coxarthrosis and gonarthrosis in clinical studies. Recommendations of the 1st Working Circle of the DGOT (Connective Tissue Research and Arthrosis Deformans)," Zeitschrift Fur Orthopadie Und Ihre Grenzgebiete, vol. 135, no. 3, pp. 193-196, 1997.

[8] P. Riegels-Nielsen, N. Frimodt-Moller, and J. S. Jensen, "Rabbit model of septic arthritis," Acta Orthopaedica Scandinavica, vol. 58, no. 1, pp. 14-19, 1987.

[9] Y. Shimizu and S. Shaw, "Lymphocyte interactions with extracellular matrix," The FASEB Journal, vol. 5, no. 9, pp. 2292-2299, 1991.

[10] C. Nathan and M. Sporn, "Cytokines in context," The Journal of Cell Biology, vol. 113, no. 5, pp. 981-986, 1991.

[11] A. D. Pearle, R. F. Warren, and S. A. Rodeo, "Basic science of articular cartilage and osteoarthritis," Clinics in Sports Medicine, vol. 24, no. 1, pp. 1-12, 2005.

[12] K. Bobacz, L. Erlacher, J. Smolen, A. Soleiman, and W. B. Graninger, "Chondrocyte number and proteoglycan synthesis in the aging and osteoarthritic human articular cartilage," Annals of the Rheumatic Diseases, vol. 63, no. 12, pp. 1618-1622, 2004.

[13] J. Jerosch, "Effects of glucosamine and chondroitin sulfate on cartilage metabolism in OA: outlook on other nutrient partners especially omega-3 fatty acids," International Journal of Rheumatology, vol. 2011, Article ID 969012, 17 pages, 2011.

[14] H. Schmal, I. H. Pilz, A. T. Mehlhorn et al., "Expression of BMPreceptor type $1 \mathrm{~A}$ correlates with progress of osteoarthritis in human knee joints with focal cartilage lesions," Cytotherapy, vol. 14, no. 7, pp. 868-876, 2012.

[15] G. M. van Buul, G. Kotek, P. A. Wielopolski et al., "Clinically translatable cell tracking and quantification by MRI in cartilage repair using superparamagnetic iron oxides," PLOS ONE, vol. 6, no. 2, Article ID el7001, 2011.

[16] M. B. Goldring, J. Birkhead, L. J. Sandell, T. Kimura, and S. M. Krane, "Interleukin 1 suppresses expression of cartilage-specific types II and IX collagens and increases types I and III collagens in human chondrocytes," The Journal of Clinical Investigation, vol. 82, no. 6, pp. 2026-2037, 1988.

[17] E. B. Hunziker, "Articular cartilage repair: are the intrinsic biological constraints undermining this process insuperable?" Osteoarthritis and Cartilage, vol. 7, no. 1, pp. 15-28, 1999.

[18] E. T. Rietschel, T. Kirikae, F. U. Schade et al., "Bacterial endotoxin: molecular relationships of structure to activity and function," The FASEB Journal, vol. 8, no. 2, pp. 217-225, 1994.

[19] M. J. Auger, "Mononuclear phagocytes. Recent research suggests a large repertoire of actions," British Medical Journal, vol. 298, no. 6673, pp. 546-548, 1989.

[20] N. V. Serbina, T. Jia, T. M. Hohl, and E. G. Pamer, "Monocytemediated defense against microbial pathogens," Annual Review of Immunology, vol. 26, pp. 421-452, 2008.

[21] M. J. Fenton, M. W. Vermeulen, B. D. Clark, A. C. Webb, and P. E. Auron, "Human pro-IL-1 $\beta$ gene expression in monocytic cells is regulated by two distinct pathways," Journal of Immunology, vol. 140, no. 7, pp. 2267-2273, 1988.

[22] J. L. Lepe-Zuniga and I. Gery, "Production of intra- and extracellular interleukin-1 (IL-1) by human monocytes," Clinical Immunology and Immunopathology, vol. 31, no. 2, pp. 222-230, 1984.

[23] H. Schmal, A. Mehlhorn, F. Stoffel, W. Kstler, N. P. Sdkamp, and P. Niemeyer, "In vivo quantification of intraarticular cytokines in knees during natural and surgically induced cartilage repair," Cytotherapy, vol. 11, no. 8, pp. 1065-1075, 2009.

[24] C. B. Knudson and W. Knudson, "Cartilage proteoglycans," Seminars in Cell and Developmental Biology, vol. 12, no. 2, pp. 69-78, 2001.

[25] K. J. Doege, M. Sasaki, T. Kimura, and Y. Yamada, "Complete coding sequence and deduced primary structure of the human cartilage large aggregating proteoglycan, aggrecan. Humanspecific repeats, and additional alternatively spliced forms," The Journal of Biological Chemistry, vol. 266, no. 2, pp. 894-902, 1991.

[26] L. S. Lohmander, M. Ionescu, H. Jugessur, and A. R. Poole, "Changes in joint cartilage aggrecan after knee injury and in osteoarthritis," Arthritis and Rheumatism, vol. 42, no. 3, pp. 534544, 1999.

[27] T. Vincent, M. Hermansson, M. Bolton, R. Wait, and J. Saklatvala, "Basic FGF mediates an immediate response of articular cartilage to mechanical injury," Proceedings of the National Academy of Sciences of the United States of America, vol. 99, no. 12, pp. 8259-8264, 2002.

[28] R. L. Sah, A. C. Chen, A. J. Grodzinsky, and S. B. Trippel, “Differential effects of bFGF and IGF-I on matrix metabolism in calf and adult bovine cartilage explants," Archives of Biochemistry and Biophysics, vol. 308, no. 1, pp. 137-147, 1994.

[29] S. Chubinskaya, M. Hurtig, and D. C. Rueger, "OP-1/BMP-7 in cartilage repair," International Orthopaedics, vol. 31, no. 6, pp. 773-781, 2007.

[30] M. Hurtig, S. Chubinskaya, J. Dickey, and D. Rueger, "BMP7 protects against progression of cartilage degeneration after impact injury," Journal of Orthopaedic Research, vol. 27, no. 5, pp. 602-611, 2009.

[31] P. H. Marks and M. L. C. Donaldson, "Inflammatory cytokine profiles associated with chondral damage in the anterior cruciate ligament-deficient knee," Arthroscopy: The Journal of Arthroscopic and Related Surgery, vol. 21, no. 11, pp. 1342-1347, 2005.

[32] F. M. Brennan, A. L. Hayes, C. J. Ciesielski, P. Green, B. M. Foxwell, and M. Feldmann, "Evidence that rheumatoid arthritis synovial T cells are similar to cytokine-activated T cells: involvement of phosphatidylinositol 3-kinase and nuclear factor kappaB pathways in tumor necrosis factor alpha production in rheumatoid arthritis," Arthritis and Rheumatism, vol. 46, no. 1, pp. 31-41, 2002.

[33] H. Schmal, A. T. Mehlhorn, J. Zwingmann, C. A. Müller, G. B. Stark, and N. P. Südkamp, "Stimulation of chondrocytes in vitro by gene transfer with plasmids coding for epidermal growth factor (hEGF) and basic fibroblast growth factor (bFGF)," Cytotherapy, vol. 7, no. 3, pp. 292-300, 2005.

[34] D. J. McQuillan, C. J. Handley, and M. A. Campbell, "Stimulation of proteoglycan biosynthesis by serum and insulinlike growth factor-I in cultured bovine articular cartilage," Biochemical Journal, vol. 240, no. 2, pp. 423-430, 1986.

[35] J. Middleton, A. Manthey, and J. Tyler, "Insulin-like growth factor (IGF) receptor, IGF-I, interleukin-1 $\beta$ (IL- $1 \beta$ ), and IL6 mRNA expression in osteoarthritic and normal human cartilage," The Journal of Histochemistry and Cytochemistry, vol. 44, no. 2, pp. 133-141, 1996.

[36] J. A. Tyler, "Insulin-like growth factor 1 can decrease degradation and promote synthesis of proteoglycan in cartilage exposed to cytokines," The Biochemical Journal, vol. 260, no. 2, pp. 543$548,1989$. 
[37] S. Chubinskaya, D. Segalite, D. Pikovsky, A. A. Hakimiyan, and D. C. Rueger, "Effects induced by BMPS in cultures of human articular chondrocytes: comparative studies," Growth Factors, vol. 26, no. 5, pp. 275-283, 2008.

[38] K. D. Huebner, N. G. Shrive, and C. B. Frank, "New surgical model of post-traumatic osteoarthritis: isolated intra-articular bone injury in the rabbit," Journal of Orthopaedic Research, vol. 31, no. 6, pp. 914-920, 2013.

[39] K. E. Kuettner and A. A. Cole, "Cartilage degeneration in different human joints," Osteoarthritis and Cartilage, vol. 13, no. 2, pp. 93-103, 2005.

[40] I. B. Mcinnes, B. P. Leung, R. D. Sturrock, M. Field, and F. Y. Liew, "Interleukin-15 mediates $\mathrm{T}$ cell-dependent regulation of tumor necrosis factor- $\alpha$ production in rheumatoid arthritis," Nature Medicine, vol. 3, no. 2, pp. 189-195, 1997.

[41] M. B. Goldring, "Osteoarthritis and cartilage: the role of cytokines," Current Rheumatology Reports, vol. 2, no. 6, pp. 459465, 2000.

[42] S. Lefèvre, A. Knedla, C. Tennie et al., "Synovial fibroblasts spread rheumatoid arthritis to unaffected joints," Nature Medicine, vol. 15, no. 12, pp. 1414-1420, 2009.

[43] H.-J. Im, P. Muddasani, V. Natarajan et al., "Basic fibroblast growth factor stimulates matrix metalloproteinase-13 via the molecular cross-talk between the mitogen-activated protein kinases and protein kinase $\mathrm{C} \delta$ pathways in human adult articular chondrocytes," Journal of Biological Chemistry, vol. 282, no. 15, pp. 11110-11121, 2007.

[44] H. Schmal, J. Zwingmann, M. Fehrenbach et al., "bFGF influences human articular chondrocyte differentiation," Cytotherapy, vol. 9, no. 2, pp. 184-193, 2007.

[45] E. W. Mandl, H. Jahr, J. L. M. Koevoet et al., "Fibroblast growth factor- 2 in serum-free medium is a potent mitogen and reduces dedifferentiation of human ear chondrocytes in monolayer culture," Matrix Biology, vol. 23, no. 4, pp. 231-241, 2004.

[46] K. Huch, "Long-term effects of osteogenic protein-1 on biosynthesis and proliferation of human articular chondrocytes," Clinical and Experimental Rheumatology, vol. 19, no. 5, pp. 525531, 2001.

[47] K. Bobacz, I. G. Sunk, J. G. Hofstaetter et al., “Toll-like receptors and chondrocytes: the lipopolysaccharide-induced decrease in cartilage matrix synthesis is dependent on the presence of tolllike receptor 4 and antagonized by bone morphogenetic protein 7," Arthritis and Rheumatism, vol. 56, no. 6, pp. 1880-1893, 2007.

[48] T. Takahashi, T. Muneta, K. Tsuji, and I. Sekiya, "BMP-7 inhibits cartilage degeneration through suppression of inflammation in rat zymosan-induced arthritis," Cell and Tissue Research, vol. 344, no. 2, pp. 321-332, 2011.

[49] S. Honsawek, M. Chayanupatkul, A. Tanavalee et al., "Relationship of plasma and synovial fluid BMP-7 with disease severity in knee osteoarthritis patients: a pilot study," International Orthopaedics, vol. 33, no. 4, pp. 1171-1175, 2009. 


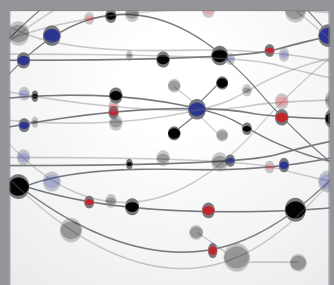

The Scientific World Journal
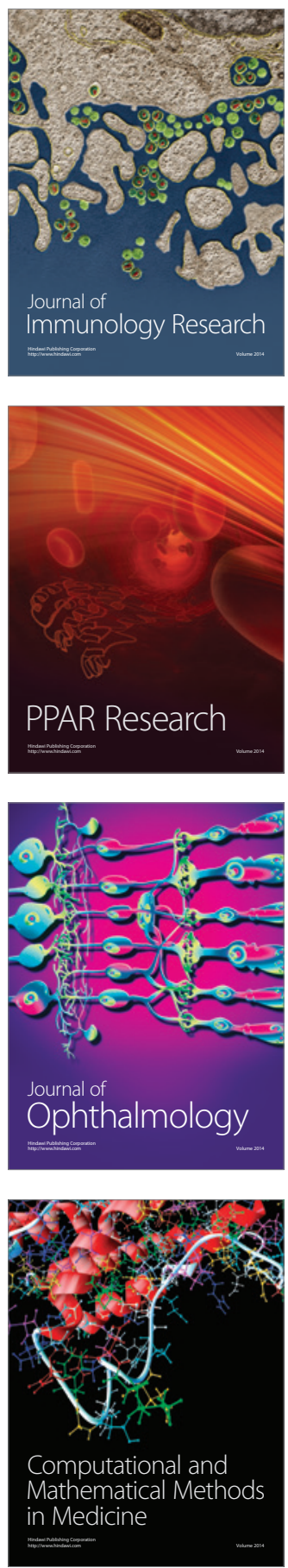

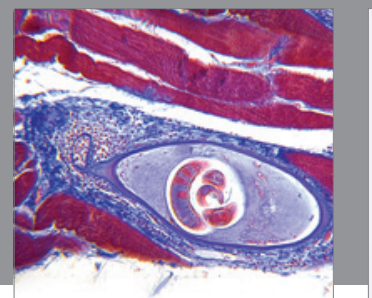

Gastroenterology

Research and Practice
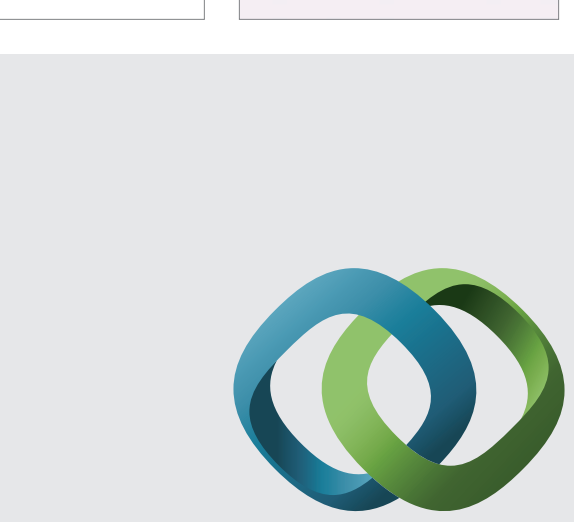

\section{Hindawi}

Submit your manuscripts at

http://www.hindawi.com
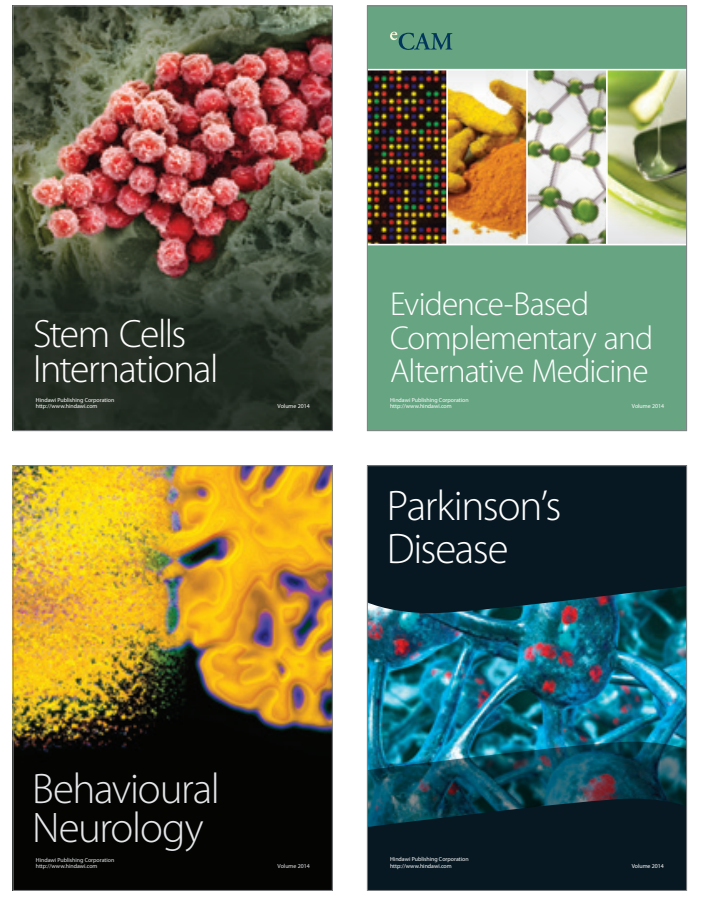
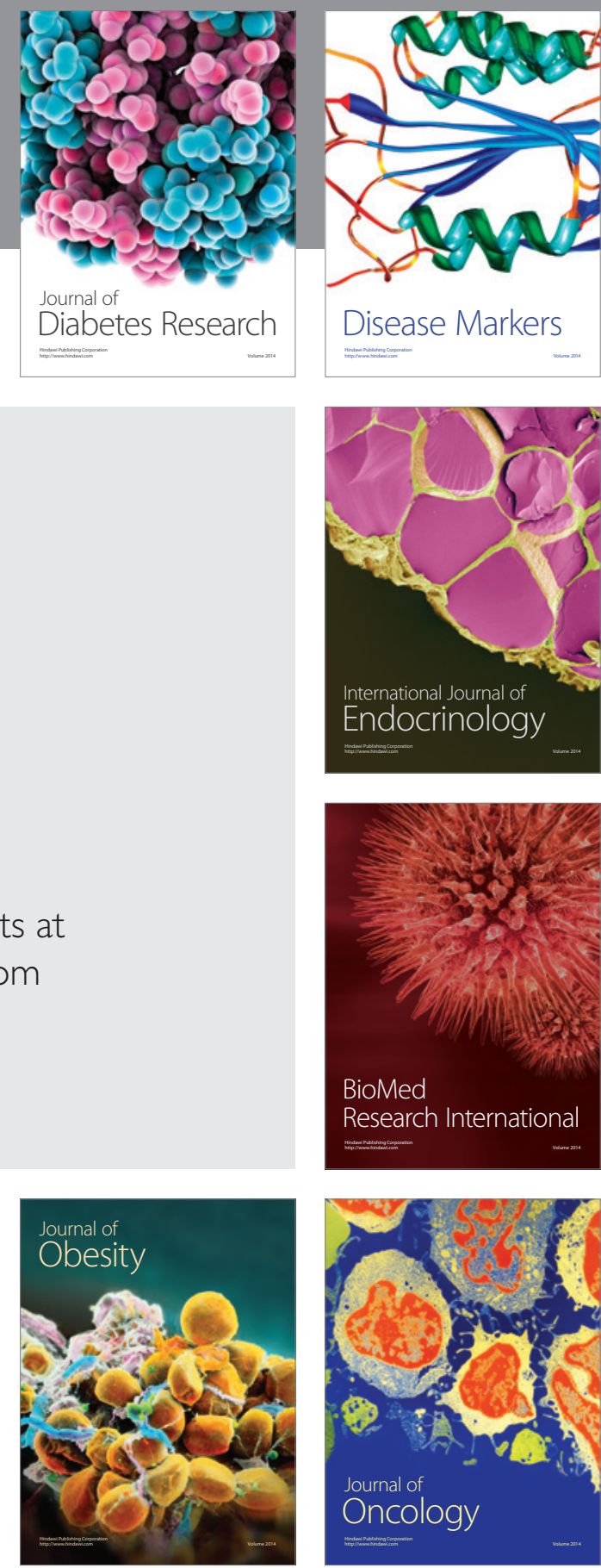

Disease Markers
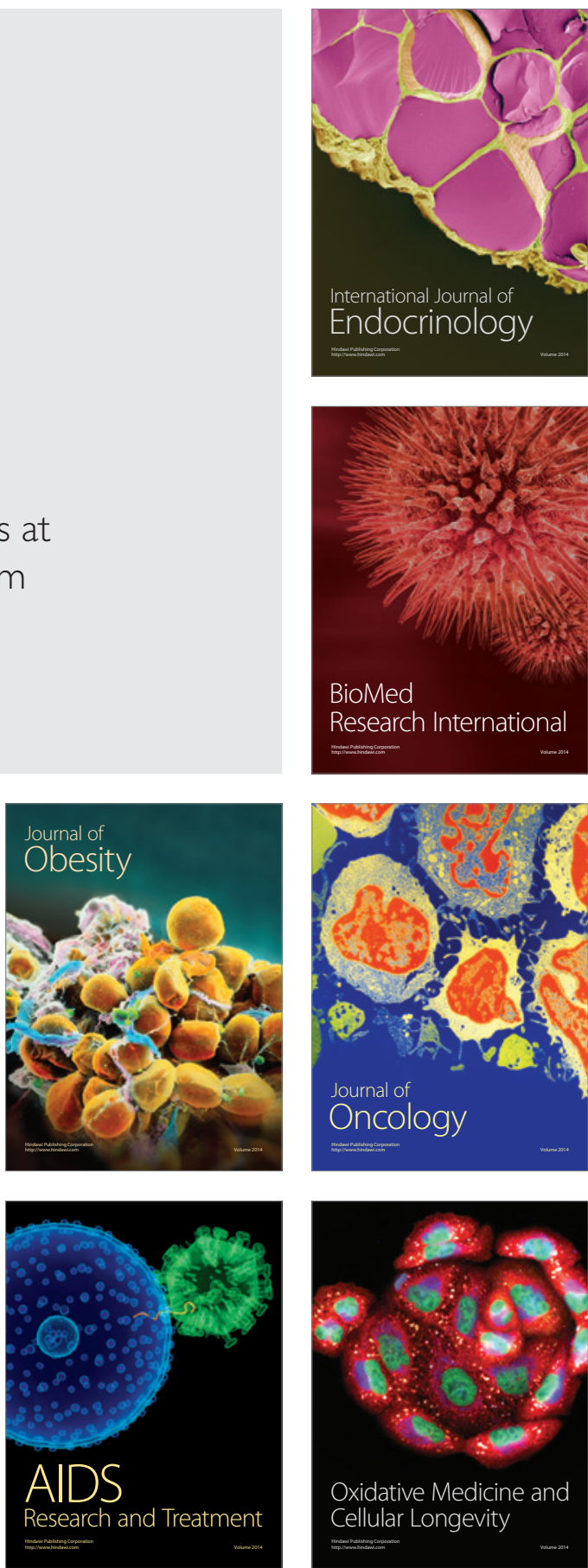